\title{
Research on Applicability of Hot-Bulb Anemometer under Low Pressure
}

\author{
Li Xiyuan ${ }^{1,2, a}$, Yin Xiaofang ${ }^{1}$, Hou Yaqin ${ }^{1}$, Gao Qinghua ${ }^{1}$ and Wang jing ${ }^{1}$ \\ ${ }^{1}$ Beijing Institute of Spacecraft Environment Engineering, Beijing 100094, China \\ ${ }^{2}$ Beihang University, Beijing 100191, China
}

\begin{abstract}
In Mars and other deep space exploration missions, the planetary atmosphere makes the difference in heat transfer characteristics on the planetary surface and on-orbit environment. In order to achieve the purposes of thermal model correction and spacecraft verification in extreme environment, Mars rover needs to be tested in a simulated Mars environment including low pressure, solar heat flux, wind speed and background temperature. Thus, wind speed should be measured at multiple points in the Mars rover thermal test. In a general Mars rover thermal balance test, the requirement for wind speed control and measurement is $0-15 \mathrm{~m} / \mathrm{s}$ under $700 \mathrm{~Pa}$ pressure. The current anemometer for industrial use is mainly based on the dynamic pressure, heat or ultrasound. They have a small signal and need to be recalibrated at low pressures. In this paper, a constant heat flux hot-bulb anemometer model has been built using dimensionless number analysis method, with which the anemometer response under low pressure has been calculated. A series of calibration test has been employed to verify the model in space environment chamber. The two methods above reached a similar result, which demonstrates the effectiveness of the analysis.
\end{abstract}

\section{Introduction}

On the surface of Mars, the atmospheric pressure is about $700 \mathrm{~Pa}$; the main component is carbon dioxide, the temperature is about $-120 \sim 20^{\circ} \mathrm{C}$, and the wind speed is $0-15 \mathrm{~m} / \mathrm{s}$. The low pressure convective makes the Mars rover face a different environment from the earth surface and the on-orbit environment. In order to achieve the purpose of thermal model correction, early failure screening, performance verification and other targets in extreme environments, low pressure, solar heat flux, wind speed and background temperature need to be simulated in a Mars rover thermal test. The thermal test of the Mars rover is usually carried out inside a large or medium space environment simulation chamber [1][2]. The cold and black background and ground contact heat transfer are achieved by the adjustable temperature shroud, the $700 \mathrm{~Pa}$ pressure is simulated by a pressure control system, and a fan is employed to blow up the wind. The wind speed in the chamber should be measured in real time across the whole test. The current anemometer for industrial use is mainly based on the dynamic pressure, heat, and ultrasound.

Dynamic pressure anemometers include wind cup, pitot tube, five-hole probe and other forms, pitot tube and five-hole probe has been widely used in low-pressure wind speed measurement, but in the $700 \mathrm{~Pa}$ environment, the dynamic pressure brought by the wind speed of $0-15 \mathrm{~m} / \mathrm{s}$ is only about $0.01 \mathrm{~Pa} \sim 10 \mathrm{~Pa}$. Although the industrial micro pressure sensor using can achieve the resolution of $10^{-}$

${ }^{a}$ Corresponding author: 1xy_422@msn.com 
${ }^{3} \mathrm{~Pa}$, they often have a larger size, heavier weight and can be hardly worked under low pressure. multipoint wind speed measurement and wind direction measurement will make the measurement system becomes hugely complicated [3].Thermal anemometers include hot wire, hot bulb, hot film and other forms, the wind speed is calculated from the sensor probe heat loss[4].Thermal anemometers are mounted on multiple Mars rovers such as the Netlander, Viking and Beagle 2. Some thermal anemometers can measure wind direction through thermal wake technique [5][6]. The thermal anemometer has the advantages of simple structure, lightweight, low power consumption, compactness and no moving parts. However, the disadvantages are also apparent: 1) calibration is crucial, and the correlation cannot be obtained by analytical model accurately; 2) As time goes on, calibration will drift slowly because of line resistance changed. 3) The change of gas temperature and pressure will also lead to the signal change. The measuring accuracy of the sensor depends on the measurement accuracy of the gas temperature and pressure. Because commercial product $\mathrm{s}$ can be hardly used in Mars environment, the present thermal anemometers used by the Mars rovers are often custom product. The ultrasonic anemometer utilizes the characteristic that the velocity of the sound wave is only related to the gas composition and temperature. Ultrasonic anemometers do not need to be calibrated under different pressure. The main drawback is that ultrasound attenuates significantly at low pressures, making it difficult to screen out signals from noise. In Mars explore, newly designed transducers are usually used instead of existing products [7][8]. Also, the ultrasonic anemometers often have a large volume, making it difficult to use in multi-point wind speed measurement.

To sum up, under the extremely low pressure, the primary method of wind speed measurement is a customized wind speed sensor at present. This paper aims at the applicability of existing industrial thermal anemometers under low pressure. The heat transfer model of the constant heat flux hot-bulb sensor under different pressure is built by the dimensionless number analysis method. The probe output under different pressures is analyzed. A test system has been developed to verify the analysis. Similar results have been obtained from the test and the dimensionless number analysis method. The correctness of the rue number analysis method has been verified, which provides a reference for the future wind speed measurement in the ground test of the Mars rovers.

\section{Convection heat transfer model of hot-bulb anemometer}

\subsection{Simulation model}

In this paper, a constant heat flux hot-bulb anemometer is selected as the research object, its diameter is $0.6 \mathrm{~mm}$, and the power is $0.08 \mathrm{~W}$. The internal structure of the probe is shown in Figure 1:

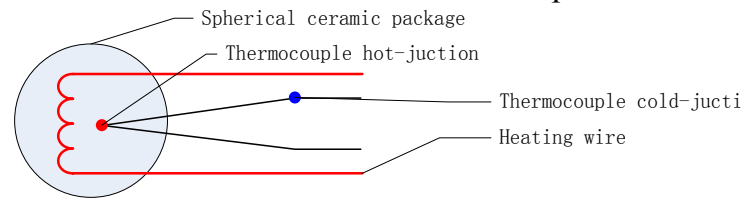

Figure 1. Probe model

When the sensor works, a constant current is applied to the heating wire to produce a constant power. In this case, the temperature of the cold junction of the thermocouple can be considered as the temperature of the fluid, and the temperature of the hot junction is hot bulb temperature. At this time, the convection heat transfer coefficient varies with the wind speed. It also leads to the difference of bulb surface temperature and difference of the thermoelectric potential. Therefore, the wind speed can be obtained by measuring the thermoelectric potential. In this paper, a customized millivolt transmitter was employed to measure the probe output.

\subsection{Dimensionless number analysis}


A convective heat transfer model for the probe has been built by dimensionless number analysis method. The external heat transfer of the probe can be divided into three forms: radiation heat transfer, convection heat transfer, and heat conduction through the probe lead and the support. In the heat balance state, The heating power is equal to the sum of the probe's heat loss:

$$
Q_{\text {Power }}=Q_{\text {con }}+Q_{\text {cond }}+Q_{\text {rad }}=k s\left(T_{s}-T_{e}\right)+h s\left(T_{s}-T_{e}\right)+\varepsilon s \sigma\left(T_{s}^{4}-T_{e}^{4}\right)
$$

Where $Q_{\text {Power }}$ is heating power on the probe $(\mathrm{W}), Q_{\text {con }}$ is the heat loss through conduction $(\mathrm{W}), Q_{\text {cond }}$ is the heat loss through convection (W), $Q_{r a d}$ is the heat loss through radiation (W), $k$ is the equivalent heat transfer coefficient for dissipating heat flow through the form of heat conduction $\left(\mathrm{W} /\left(\mathrm{m}^{2} \bullet \mathrm{K}\right)\right), S$ is the surface area of the hot bulb $\left(\mathrm{m}^{2}\right), h$ is the convection heat transfer coefficient $\left(\mathrm{W} /\left(\mathrm{m}^{2} \cdot \mathrm{K}\right)\right), \varepsilon$ is the emissivity of the hot bulb surface, $\sigma$ is Stefan-Boltzmann's constant, and the value is $5.67 \times 10^{-8} \mathrm{~W} /\left(\mathrm{m}^{2} \cdot \mathrm{K}^{4}\right), T_{s}$ is the temperature of the hot bulb, $T_{e}$ is the temperature of the gas. When conduction and radiation heat transfer is negligible, the equation can be simplified as follow:

$$
C_{1} Q_{\text {Sensor }}=h s\left(T_{s}-T_{e}\right)
$$

Where $Q_{\text {Sensor }}$ is the total heat flow on the anemometer, $C_{1}$ is the ratio of heat flow on the hot bulb to the total heat flow. Reynolds number near the hot bulb probe can be calculated as:

$$
R e=\frac{v l}{v}=\frac{v 1 \rho}{\mu}
$$

Where $V$ is gas flow velocity $(\mathrm{m} / \mathrm{s}), 1$ is the characteristic length of the hot bulb(m), its value is $0.0006 \mathrm{~m}, v$ is the kinematic viscosity of the gas $\left(\mathrm{m}^{2} / \mathrm{s}\right)$, can be calculated by dynamic viscosity $\mu$ $(\mathrm{Pa} \cdot \mathrm{S}) \cdot$ and the density $\rho\left(\mathrm{kg} / \mathrm{m}^{3}\right)$. The dynamic viscosity does not vary with the density of the gas, and the density can be calculated through ideal gas formula by gas pressure and temperature.

According to the calculation, when the wind speed is among $0-15 \mathrm{~m} / \mathrm{s}$ under ambient pressure, the Reynolds number is about 20 500, while in the 700Pa environment, the Reynolds number is only 0.1 $\sim 2$ when the wind speed is among $0-15 \mathrm{~m} / \mathrm{s}$. There are a variety of empirical formulas that give the relationship between Reynolds and Nusselt numbers. Kramers, Whitaker, Yuge, Vilet, and Raithby [9] have presented their expressions for different ranges, but most of them are suitable for medium and high Reynolds numbers. For Reynolds number among 0.1-100, most of the formulas have more than $50 \%$ error. The Whitaker correlation is closest to the experimental result at low Reynolds number[10]:

$$
N u=2+\left(0.4 \operatorname{Re}^{0.5}+0.06 \operatorname{Re}^{2 / 3}\right) \operatorname{Pr}^{0.4}
$$

Where $P r$ is Prandtl number. Convection heat transfer coefficient $\mathrm{h}$ can be expressed by Nusselt number:

$$
h=\frac{N u \lambda}{1}
$$

Where $\lambda$ is thermal conductivity $(\mathrm{W} /(\mathrm{m} \cdot \mathrm{K}))$. It changes little when the gas pressure changes. Therefore, the temperature difference between the sphere and the incoming gas can be expressed as:

$$
\Delta T=\frac{C_{1} Q_{\text {Sensor }} l}{S f_{\lambda}\left(T_{\mathrm{e}}+\Delta T\right)\left(2+\left(0.4 \times\left(\frac{v l \rho}{f_{\mu}\left(T_{\mathrm{e}}+\Delta T\right)}\right)^{0.5}+0.06 \times\left(\frac{v l \rho}{f_{\mu}\left(T_{\mathrm{e}}+\Delta T\right)^{0.5}}\right)_{P_{r}}\left(T_{\mathrm{e}}+\Delta T\right)^{0.4}\right)\right.}
$$

Where $T_{\mathrm{e}}$ is incoming gas temperature $\left({ }^{\circ} \mathrm{C}\right), f_{\lambda}, f_{\mu}, f_{P_{Y}}$ are functions of physical parameters change with temperature, respectively. When considering the thermocouple output signal and the temperature was linear, the above equation can be simplified as: 


$$
\Delta u=\frac{C Q_{\text {Sensor }} 1}{S f_{\lambda}\left(T_{\mathrm{e}}+\Delta T\right)\left(2+\left(0.4 \times\left(\frac{v 1 \rho}{f_{\mu}\left(T_{\mathrm{e}}+\Delta T\right)}\right)^{0.5}+0.06 \times\left(\frac{v 1 \rho}{f_{\mu}\left(T_{\mathrm{e}}+\Delta T\right)}\right)^{0.5}\right) f_{P_{r}}\left(T_{\mathrm{e}}+\Delta T\right)^{0.4}\right)}
$$

Where constant $C$ includes the thermocouple temperature coefficient and constant $C_{1}$, the surface area error, a small amount of radiation, thermal conductivity and other parameters that difficult to obtain by analysis are also embodied in the constant. The constant $C$ can only be solved by the experimental method.

\subsection{Natural Convection and Forced Convection Intensity Analysis}

There are both natural convection and forced convection around the thermal anemometer probe. For a constant heat flux probe, natural convection intensity also changes with the probe surface temperature. In a wind measurement test, the force convection should be the primary heat transfer forms around the probe to minimize the errors caused by natural convection. The intensity of natural convection and forced convection can be evaluated by dimensionless number $\mathrm{Gr} / \mathrm{Re}^{2}$ :

$$
\frac{G r}{\operatorname{Re}^{2}}=\frac{\frac{g a_{v} \Delta t 1^{3}}{v^{2}}}{\left(\frac{v l}{v^{2}}\right)^{2}}=\frac{g a_{v} \Delta t 1}{v^{2}}
$$

Where $G r$ is Grashov number, $g$ is the gravitational acceleration $\left(\mathrm{m} / \mathrm{s}^{2}\right), \Delta t$ is the temperature difference between the hot bulb and the ambient gas, $a_{v}$ is the body expansion coefficient $\left(\mathrm{K}^{-1}\right)$, can be expressed as follows:

$$
a_{V}=-\frac{1}{\rho}\left(\frac{\partial \rho}{\partial T}\right)_{p}=-\frac{1}{\rho}\left(\frac{\partial\left(\frac{P}{R T}\right)}{\partial T}\right)_{p}=-\frac{1}{\rho} \times \frac{P}{R} \times-\frac{1}{T^{2}}=\frac{1}{T}
$$

Where $\rho$ is $\operatorname{density}\left(\mathrm{kg} / \mathrm{m}^{3}\right), P$ is pressure $(\mathrm{Pa}), T$ is temperature $(\mathrm{K}), R$ is the gas constant. As the formula shows, body expansion coefficient $a_{V}$ is independent of pressure. Therefore, the $G r / R e^{2}$ is only related to gravitational acceleration, characteristic temperature, temperature difference, characteristic length and flow velocity, of which gravitational acceleration, and characteristic length and flow velocity are set value, temperature difference can be calculated by equ.5. Characteristic temperature is the average temperature of the incoming gas and the temperature of the hot bulb. The flow is dominated by force convection when $G r / R e^{2}$ is below 0.01 , is dominated by natural convection when $G r / R e^{2}$ is higher than 10. When $G r / R e^{2}$ is between 0.01 and 10 , both force convection and natural convection should be considered in the analysis.

\section{Test}

According to the literature [3], there are three basic methods for simulating the wind speed at a low pressure of about $1000 \mathrm{~Pa}$, the rotation method, the closed wind tunnel method, and the open wind tunnel method. In the rotation method, the rotating platform drives the wind probe to rotate, the fluid medium is not moving, and the relative motion between the probe and the fluid medium is the wind speed. The fundamental principle is shown in Figure 2. In general, the cantilever and the rotating platform are set in a space environment simulation chamber, and the chamber is filled with the gas with desired pressure and temperature. The rotation method has the advantages of low cost, simple structure, pressure and gas composition stable. In order to verify the dimensionless number analysis 
model, a rotating calibration system has been built based on a median-size space environment simulation chamber, as shown in Figure 2:
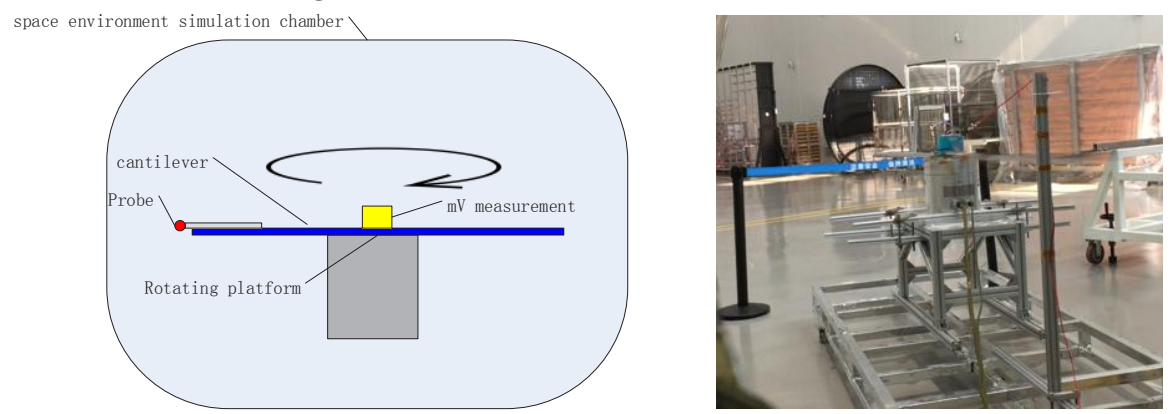

Figure 2. Rotating wind speed simulation system

As can be seen from the figure above, the hot bulb probe is fixed on the end of the cantilever. Wind speed around the probe can be calculated by the rotating platform angle speed and length of the cantilever. The 12-bit precision transmitter measures the millivolt signal and transfers the data to the computer outside the chamber by RS485. It is set on the rotating platform and can work in vacuum environment directly. The acquisition resolution is $0.01 \mathrm{mv}$. The probe's constant heat flow was applied by a programmable power outside the chamber, the total power of the anemometer probe was $0.08 \mathrm{~W}$. In this paper. The probe was tested in space environment chamber under ambient pressure, $40000 \mathrm{~Pa}$, and $700 \mathrm{~Pa}$ and the background and fluid temperature was ambient temperature. The output signal under ambient pressure is compared with the signal predicted by the dimensionless number analysis method, as shown in Figure $3(C \approx 36.10), G r / R^{2}$ under different wind speed was shown in Figure 4. Because of the aerodynamic drag under ambient pressure, the maximum simulated line speed is $11 \mathrm{~m} / \mathrm{s}$.

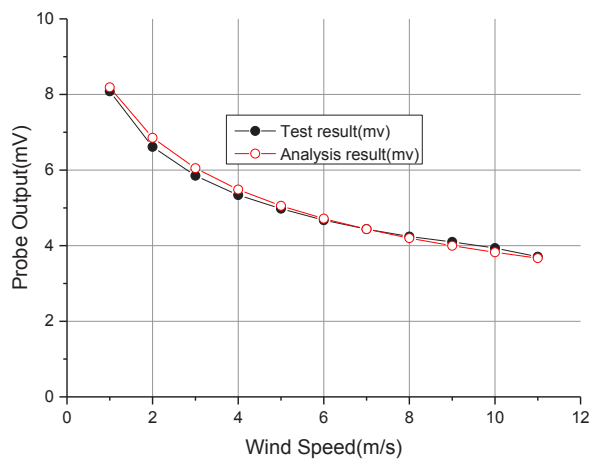

Figure 3. Probe output and dimensionless number analysis result under ambient pressure

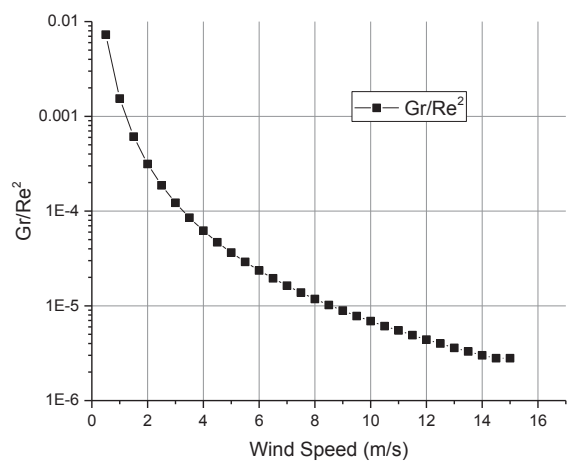

Figure 4. $G r / R e^{2}$ at different winds speed under ambient pressure

As can be seen from the figure, under the ambient pressure, the dimensionless number analysis results were in good agreement with experimental data under ambient pressure. The $\mathrm{Gr} / \mathrm{Re}^{2}$ were below 0.01 in all test case, so that the force convection occupied a dominant position in the convection around the hot bulb probe. The probe output and analysis result under $40000 \mathrm{~Pa}$ and $700 \mathrm{~Pa}$ are shown in Figure 5. $G r / R^{2}$ under 700Pa are shown in Figure 6: 


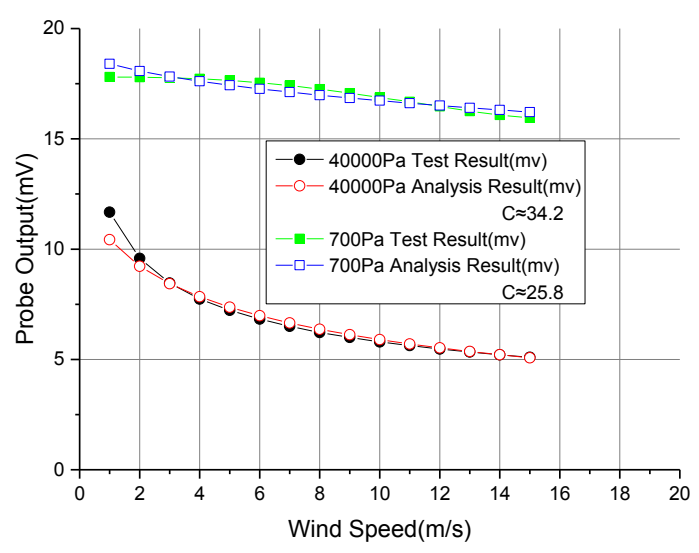

Figure 5. Probe output and analysis result under $40000 \mathrm{~Pa}$ and $700 \mathrm{~Pa}$

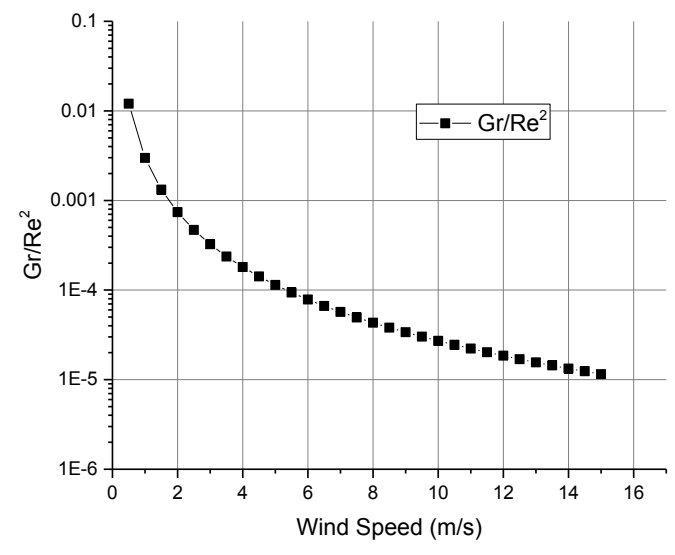

Figure 6. $G r / R e^{2}$ at different winds speed under $700 \mathrm{~Pa}$ pressure

As can be seen in Figure 6, Gr/Re $e^{2}$ is still less than 0.01 for the wind speed above $1 \mathrm{~m} / \mathrm{s}$. Therefore, the forced convection still occupies the dominant position in the total convective heat transfer, and natural convection will not bring additional measurement error. As the output signal and analysis result are seen in Figure 5, the dimensionless number analysis can be consistent with the test results by modifying the constant $\mathrm{C}$. The constant $\mathrm{C}$ is about 0.041 at $40000 \mathrm{~Pa}$, and it deviates $7.1 \%$ from the ambient pressure result, but, the deviation is more than $44.9 \%$ under $700 \mathrm{~Pa}$ pressure. The change is mainly due to the change of $\mathrm{Nu}-\mathrm{Re}$ correlation with pressure. This result is similar to the experimental and analytical results in [11]. Thus, anemometers probe using under low pressure should be corrected with the experimental data. Meanwhile, because of the background temperature dependence of the radiation heat transfer, when using constant heat flow hot bulb anemometer under low pressure, the calibration environment should have similar radiation background, or radiation insulation material should be set around the probe to minimize the errors caused by radiation heat transfer. Also, as shown in Figure 6, the sensitivity of the sensor at different wind speeds is about $0.01-0.2 \mathrm{mv} /\left(\mathrm{m} \cdot \mathrm{s}^{-1}\right)$ under $700 \mathrm{~Pa}$. When the wind speed measurement is performed at a low pressure, the millivolt signal data transmitter should also meet the accuracy requirements.

\section{Conclusion}

Aiming at the problem of wind speed measurement under low pressure, this paper establishes a model of wind speed and output signal for industrial hot-bulb anemometer by using the dimensionless number analysis method. A rotating low-pressure wind speed calibration system has been built based on median size space environment simulation chamber. The system has been tested under ambient pressure, $40000 \mathrm{~Pa}$ and $700 \mathrm{~Pa}$ respectively. The sensor output under different wind speed conditions was obtained. The dimensionless number analysis model was corrected by the experimental data. The simulation model and the experiment achieved a similar result. In this paper, the dimensionless number analysis model for constant heat flow hot bulb probe can be directly applied to the output signal prediction at a pressure above $40000 \mathrm{~Pa}$ and can be corrected by experimental data for $700 \mathrm{~Pa}$ low-pressure environment. The analysis method can be used to evaluate the output of different anemometers in different environments, and provide a reference for the development of the corresponding test in future.

\section{References}


1. Ransome T, Pesket S, Toplis G. Thermal Balance Testing of the Beagle2 Mars Lander[C]. International Symposium Environmental Testing for Space Programmes, 2001.

2. Johnson K. Simulation of Mars Surface conditions for Characterization of the Mars Rover Thermal Response[J].

3. Wilson C F. Measurement of wind on the surface of Mars[D]. Oxford, 2003.

4. Bruun H H. Hot-Wire Anemometry[J]. Oxford Univ Pr, 1995.

5. Chamberlain T E, Cole H L, Dutton R G, et al. Atmospheric measurements on Mars - The Viking meteorology experiment[J]. Bulletin of the American Meteorological Society, 1976, 57(9).

6. Seiff A, Tillman J E, Murphy J R, et al. The atmosphere structure and meteorology instrument on the Mars Pathfinder lander[J]. Journal of Geophysical Research Planets, 1997, 102(E2): 40454056 .

7. Kapartis S S R. Wind speed and direction measurement using Acoustic Resonance airflow sensing. FT Technologies, 1999.

8. Banfield D, Gierasch P J, Toigo A, et al. Mars Acoustic Anemometer[J], 2012.

9. Will J B, Kruyt N P, Venner C H. An experimental study of forced convective heat transfer from smooth, solid spheres[J]. International Journal of Heat \& Mass Transfer, 2017, 109:1059-1067.

10. Dennis S C R, Walker J D A, Hudson J D. Heat transfer from a sphere at low Reynolds numbers[J]. Journal of Fluid Mechanics, 2006, 60(2):273-283.

11. Numata D, Anyoji M, Sugino Y, et al. Characteristics of Thermal Anemometers at Low-Pressure Condition in a Mars Wind Tunnel[C]// Aiaa Aerospace Sciences Meeting Including the New Horizons Forum and Aerospace Exposition. 2013. 